\title{
Implementation of a closed-loop structural control system using wireless sensor networks
}

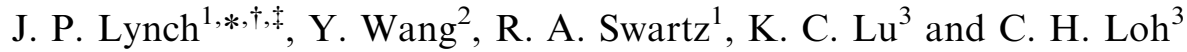 \\ ${ }^{1}$ Department of Civil and Environmental Engineering, University of Michigan, Ann Arbor, MI 48109-2125, U.S.A. \\ ${ }^{2}$ Department of Civil and Environmental Engineering, Stanford University, Stanford, CA 94305, U.S.A. \\ ${ }^{3}$ Department of Civil Engineering, National Taiwan University, Taipei, Taiwan
}

\begin{abstract}
SUMMARY
Wireless sensor networks have rapidly matured in recent years to offer data acquisition capabilities on par with those of traditional tethered data acquisition systems. Entire structural monitoring systems assembled from wireless sensors have proven to be low cost, easy to install, and accurate. However, the functionality of wireless sensors can be further extended to include actuation capabilities. Wireless sensors capable of actuating a structure could serve as building blocks of future generations of structural control systems. In this study, a wireless sensor prototype capable of data acquisition, computational analysis and actuation is proposed for use in a real-time structural control system. The performance of a wireless control system is illustrated using a full-scale structure controlled by a semi-active magnetorheological (MR) damper and a network of wireless sensors. One wireless sensor designated as a controller automates the task of collecting state data, calculating control forces, and issuing commands to the MR damper, all in real time. Additional wireless sensors are installed to measure the acceleration and velocity response of each system degree of freedom. Base motion is applied to the structure to simulate seismic excitations while the wireless control system mitigates inter-storey drift response of the structure. An optimal linear quadratic regulation solution is formulated for embedment within the computational cores of the wireless sensors. Copyright (C) 2007 John Wiley \& Sons, Ltd.
\end{abstract}

KEY WORDS: structural control; wireless sensors; embedded computing; magnetorheological dampers

\footnotetext{
*Correspondence to: J. P. Lynch, Department of Civil and Environmental Engineering, University of Michigan, Ann Arbor, MI 48109-2125, U.S.A.

†E-mail: jerlynch@umich.edu

Assistant Professor

Contract/grant sponsor: Office of Naval Research (ONR)

Contract/grant sponsor: National Centre for Research on Earthquake Engineering (NCREE)

Contract/grant sponsor: National Science Council of Taiwan
}

Copyright (C) 2007 John Wiley \& Sons, Ltd.

Received 17 May 2006

Revised 16 March 2007

Accepted 3 April 2007 


\section{INTRODUCTION}

Recent natural catastrophes have revealed the vulnerabilities of critical civil infrastructure systems (bridges, buildings, tunnels, dams) exposed to earthquakes, hurricanes, and typhoons. To mitigate structural responses resulting from dynamic loads, feedback control systems have been proposed by Yao [1] for installation in civil structures. Since that time, various world conferences [2-4], regional workshops [5], and publications [6-8] dedicated to structural control have rapidly advanced the state of the art. As a result, feedback control systems have been widely adopted with over 50 buildings and 20 long-span bridges in Asia currently employing feedback control [9]. Early structural control systems proposed for civil structures employed large actuators for the direct application of control forces. While active control systems were successful at mitigating structural responses to wind loads, force capacities of actuators often saturate during large seismic events, thereby limiting their effectiveness. In response to this limitation, the concept of semi-active structural control was proposed. Unlike active actuators, semi-active control devices are designed to develop internal structural forces by changes to the damping and stiffness properties of the structure [7]. Examples of semi-active devices include, but are not limited to: active variable stiffness (AVS) devices [10], semi-active hydraulic dampers (SHD) [11], electrorheological (ER) dampers [12], and magnetorheological (MR) dampers [13]. A benefit of developing control forces in a structure indirectly is that semi-active control devices consume an order of magnitude less power than actuators associated with active control systems [14]. In addition to inherent energy efficiencies, semi-active control devices are compact and low cost. These attractive attributes encourage the use of large numbers of semi-active devices in a structure; examples include 88 SHD devices in the Shiodome Tower, Tokyo and over 350 SHD devices in the Mori Tower, Tokyo [9].

As recent installations suggest, future semi-active control systems will continue to be defined by ever greater nodal densities. As structural control systems grow in size, design and installation complexities increase in tandem. For example, structural control systems currently employ extensive lengths of coaxial wire to accommodate communication between sensors, actuators, and a centralized controller. As nodal densities increase, more coaxial wire is needed for communication. In 2002, the installation of coaxial wire between sensors and a central data repository has been cited to cost as high as a few thousand dollars per sensor channel [15]. As a result, the benefit derived from additional control devices are eroded by the high installation costs associated with increasing lengths of coaxial wire. To eradicate the high cost of a wired control system, the use of wireless communications is proposed for systems defined by high nodal densities.

Other researchers have previously explored wireless communications for adoption in feedback control systems. Unlike traditional control systems that have dedicated coaxial wires between sensors, actuators and the centralized controller, a control system adopting wireless communications requires sensors and controllers to share a common wireless medium for communication. When a closed-loop control system is implemented using a common communication medium (wired or wireless), network quality strongly influences the performance of the control solution. Specifically, time delays governed by deterministic and stochastic processes are often introduced by the network. Lian et al. [16] propose the use of network protocols that guarantee deterministic transmission times between transmitting and receiving nodes so that delays can be accounted for by the control solution. However, stochastic delays sometimes cannot be avoided and are difficult to account for a priori. Specific to wireless 
networks, multiple researchers have begun to explore real-time closed-loop control using wireless sensors. Eker et al. [17] explores the implementation of a linear quadratic regulation (LQR) control solution using a wireless controller that communicates using the Bluetooth wireless communication protocol. Randomly varying delays within the wireless communication channel are compensated for in the design of the LQR controller using a compensation technique proposed by Nilsson et al. [18]. Ploplys et al. [19] implements a closed-loop control solution for an inverted pendulum using a wireless sensor network communicating upon the IEEE $802.11 \mathrm{~b}$ communication standard. To ensure timely delivery of data packets, the User Datagram Protocol (UDP) is adopted to provide fast sample rates and to reduce network congestion. In recent years, various researchers in the structural control community have also explored wireless control systems. For example, Casciati and Rossi [20] propose the use of wireless sensors with embedded fuzzy chip controllers; their work validates the concept using laboratory experiments. In addition, Lynch [21] reports on the design of wireless sensors capable of actuating structural control actuators.

In this study, a real-time structural control system for civil structures is advanced using wireless sensor networks. As a fundamental building block of the control system, a wireless sensor prototype is designed to provide the functionality required for real-time control including data collection, computation, and actuation. The hardware design of the wireless sensor described herein is largely based upon a wireless sensor previously proposed for infrastructure monitoring [22]. The actuation interface of the modified wireless sensor is designed to output an analog voltage signal to command semi-active control devices in real time. One challenge associated with wireless communications is the reliable delivery of data in the network. To address this challenge, a wireless communication protocol is proposed based upon a time division multiple access (TDMA) communication scheme. The feasibility of a wireless control system is validated using a full-scale three-storey steel structure excited by seismic ground motions. A $20 \mathrm{kN}$ MR damper is installed at the base of the structure for mitigation of structural responses (specifically inter-storey drifts). The bounded input-bounded output (BIBO) stability properties of semi-active dampers protect the test structure from becoming unstable should the wireless control system perform poorly. Two control system architectures are implemented; one architecture adopts velocity transducers while the second adopts accelerometers. The performance of the wireless control system will be quantified by comparing the closed-loop control performance to utilizing the MR damper in a passive configuration. In addition, the wireless control system performance is also compared to that of a control system implemented using a wired laboratory data acquisition system. As a result of computational and communication overhead, the wireless control system is operated at a $12.5 \mathrm{~Hz}$ sample rate while the baseline tethered control system operates at $200 \mathrm{~Hz}$. The wireless control system is shown to be both effective in reducing structural responses and reliable in the wireless delivery of state data at each time step.

\section{PROTOTYPE WIRELESS SENSOR FOR MONITORING AND CONTROL}

Numerous commercial and academic wireless sensors have been proposed for monitoring civil structures [23]. The hardware design of all of these wireless sensors can be divided into four functional components: sensing interface, computational core, wireless communication channel, and actuation interface. A large majority of the wireless sensors proposed for structural 


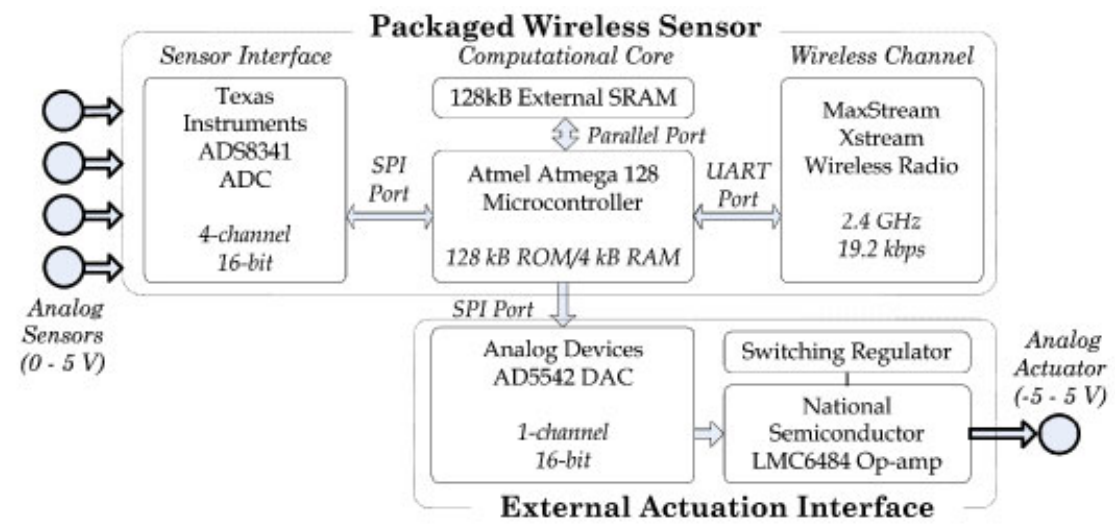

(a)

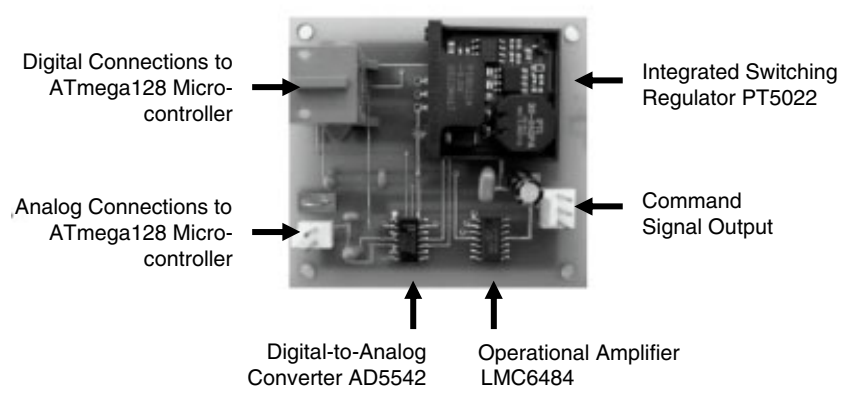

(b)

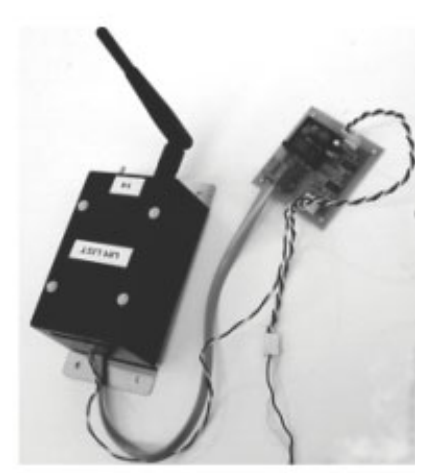

(c)

Figure 1. (a) Architectural overview of a wireless sensor prototype for structural control; (b) stand-alone actuation interface circuit; and (c) fully assembled wireless sensor with external actuation interface attached.

monitoring integrate the first three functional components. However, recent wireless sensor designs have begun to include an actuation interface as a fourth functional component [21]. This study will modify the design of an existing wireless sensor prototype to accommodate an actuation interface for use in a control system [22]. The design of the entire wireless sensor prototype is presented in Figure 1(a) while the design of the actuation interface is described in greater detail below.

The actuation interface is housed upon its own two-layer printed circuit board that is externally attached to the main wireless sensor package. The major hardware component integrated in the actuation interface is a digital-to-analog converter (DAC) which receives binary numbers from the wireless sensor microcontroller and converts them to zero-order hold $(\mathrm{ZOH})$ analog voltage signals. The single-channel 16-bit Analog Devices AD5542 DAC, capable of a maximum sample rate of $1 \mathrm{MHz}$, is selected. The wireless sensor provides a $5 \mathrm{~V}$ power supply for the AD5542, effectively allowing the DAC to output analog voltage signals from 0 to $5 \mathrm{~V}$. An additional operational amplifier (National Semiconductor LMC6484) is included to broaden the $0-5 \mathrm{~V}$ output range of the DAC to -5 to $5 \mathrm{~V}$. To provide negative voltage outputs, 
the op-amp must be powered with a 5 and $-5 \mathrm{~V}$ voltage supply. The $5 \mathrm{~V}$ supply is provided by the wireless sensor; the $-5 \mathrm{~V}$ supply is generated by a Texas Instruments PT5022 switching regulator which converts the regulated $5 \mathrm{~V}$ power supply of the wireless sensor to a $-5 \mathrm{~V}$ supply. Figure 1(b) provides a picture of the stand-alone actuation interface circuit with its main hardware components highlighted.

The fully assembled wireless sensor pictured in Figure 1(c) is packaged in a compact container $\left(6.4 \times 10 \times 8 \mathrm{~cm}^{3}\right)$ with the actuation interface externally connected using a series of wires carrying power and electrical signals. To power the completed prototype, $5 \mathrm{AA}$ lithium-ion batteries are included in the hardened container. The sensing interface and computational core consumes $32 \mathrm{~mA}$ of electrical current when powered by the regulated $5 \mathrm{~V}$ battery source. Similarly, the stand-alone actuation interface consumes $5 \mathrm{~mA}$ of current when referenced at $5 \mathrm{~V}$. It should be noted that the actuation interface is not intended to power an actual actuator, but rather, to issue low-power command signals to an independently-powered actuator. In contrast to the low power demands of the sensor interface, actuation interface and computational core, the wireless modem (Maxstream 24XStream) consumes 150, $80 \mathrm{~mA}$ and $26 \mu \mathrm{A}$ of current when transmitting, receiving and in standing-by, respectively. If it is assumed that the wireless modem is transmitting and receiving in equal proportion, the operational life expectancy of the wireless sensor is roughly $20 \mathrm{~h}$. For control applications, this represents sufficient life expectancy since the system is triggered on to operate over the duration of a single earthquake which lasts only a few minutes.

\section{SEMI-ACTIVELY CONTROLLED STEEL STRUCTURE}

\subsection{Full-scale validation structure excited by base motions}

In this study, a full-scale steel structure (Figure 2) is constructed upon a large shaking table (5 by $5 \mathrm{~m}^{2}$ footprint) at the National Centre for Research on Earthquake Engineering (NCREE), Taiwan. The shaking table is commanded in 6 degrees of freedom so that a realistic seismic loading can be applied to the base of the structure. The shaking table is capable of exerting motions between 0.1 and $50 \mathrm{~Hz}$ with a maximum acceleration and inertial force of $9.8 \mathrm{~m} / \mathrm{s}^{2}$ and $220 \mathrm{kN}$, respectively. The steel structure is a single bay, three-storey building frame constructed from I-beam $(\mathrm{H} 150 \times 150 \times 7 \times 10)$ steel elements. The height of each floor is $3 \mathrm{~m}$ (total structure height is $9 \mathrm{~m}$ ) and the floor area is 3 by $2 \mathrm{~m}^{2}$. The floors are designed as rigid diaphragms that do not deform when the structure is excited by base motion; rather, the lateral response of the structure is through shear deformation of the four columns. Additional mass is applied to each floor to ensure a total mass of $6000 \mathrm{~kg}$ is associated with each lateral degree of freedom. Experimental testing on the structure reveals the structure to be lightly damped with a damping ratio of roughly $3 \%$.

\subsection{Semi-active magnetorheological damper}

An MR damper, whose damping coefficient can be changed in real time, is installed at the base of the steel structure. The damper is installed within a steel V-brace to transfer the damper force to the first floor of the structure. The MR damper is capable of a maximum control force of $20 \mathrm{kN}$, stroke of $10.8 \mathrm{~cm}$, and can be controlled by a simple command voltage ranging from 0 to $1 \mathrm{~V}$ [24]. To power the MR damper, a separate $24 \mathrm{~V}$ laboratory power supply is employed; the 


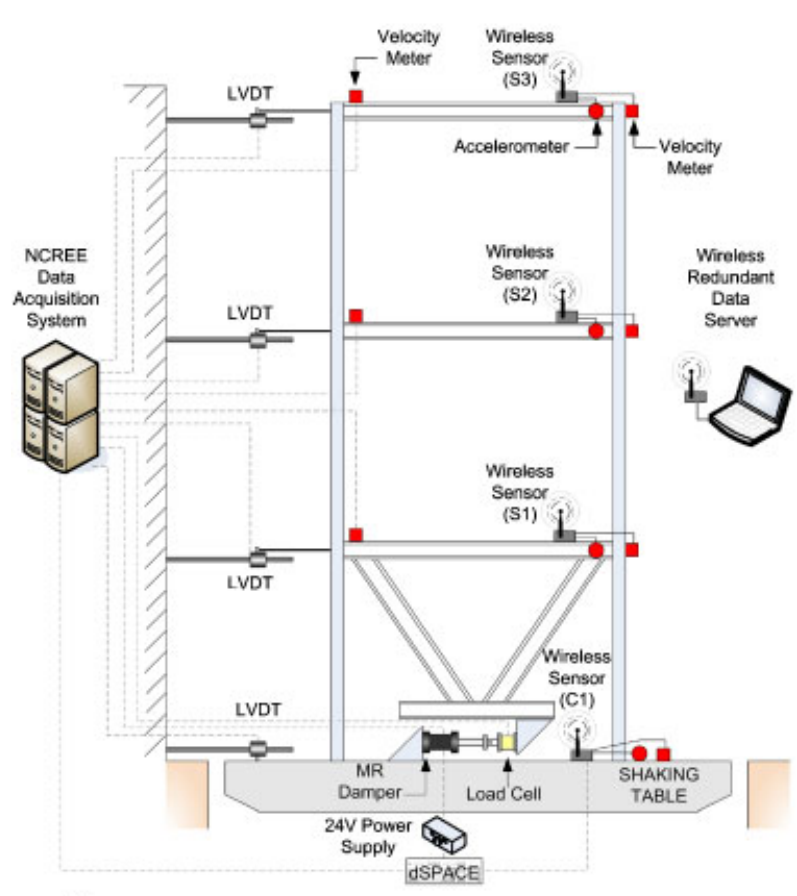

(a)

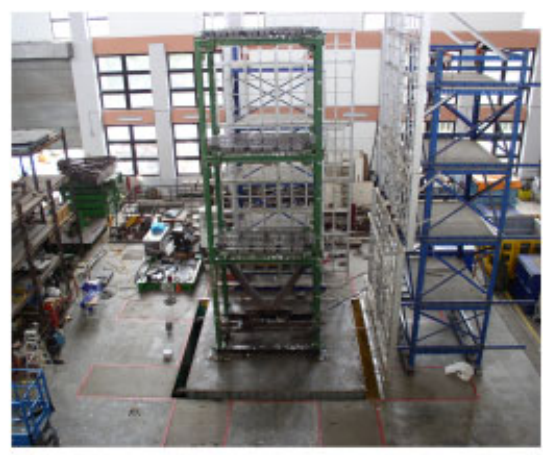

(b)

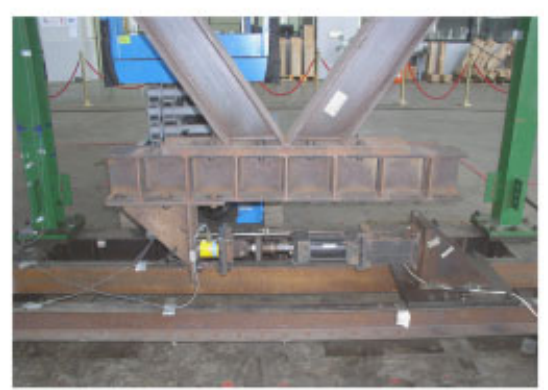

(c)

Figure 2. (a) Overview of the three-storey test structure and its instrumentation; (b) completed structure mounted to the NCREE shaking table; and (c) close-up view of the MR damper and load cell at the structure base.

power supply receives the 0 to $1 \mathrm{~V}$ command signal and converts the voltage command signal to an electrical current ( 0 to $2 \mathrm{~A})$ that is then delivered to the damper coil. The MR damper, including a load cell installed in series, is pictured in Figure 2(c).

MR dampers are nonlinear devices that must be properly modelled before they can be employed within a structural control system. A number of parametric models that fully describe the force-velocity relationships of MR dampers have been formulated [13]. One such parametric model is the Bouc-Wen model, whose computational tractability and model flexibility are attractive features. For the $20 \mathrm{kN}$ MR damper used in this study, a modified Bouc-Wen model has been proposed by Lin et al. [24]. The force in the MR damper, $F$, results from an equivalent viscous damper with the addition of a hysteretic restoring force, $z$ :

$$
F(t)=C(V) \dot{x}(t)+z(t)
$$

Here, the damping coefficient, $C$, is controllable by the damper command voltage, $V$. In this study, the hysteretic restoring force, $z$, is defined by a modified Bouc-Wen model $[25,26]$,

$$
\dot{z}(t)=A \dot{x}(t)+\sum_{n=1}^{2} a_{n}\left[\gamma \dot{x}(t)|z(t)|^{n}+\beta|\dot{x}(t)||z(t)|^{n-1} z(t)\right]
$$

where, $A, \beta, \gamma$, and $n$ are parametric constants and $\dot{x}(t)$ is the shaft velocity of the damper. The modified Bouc-Wen model can be written in discrete time at time step, $k$ 
(where $t=k \Delta t$ ):

$$
\begin{aligned}
& z(k)-z(k-1)=\Delta t\left[\boldsymbol{\Phi}(k-1)^{\mathrm{T}} \boldsymbol{\Theta}\right] \\
& \boldsymbol{\Theta}^{\mathrm{T}}=\left\{\begin{array}{lllll}
\theta_{1} & \theta_{2} & \theta_{3} & \theta_{4} & \theta_{5}
\end{array}\right\} \\
& \boldsymbol{\Phi}(k)^{\mathrm{T}}=\left\{\dot{x}(k)|\dot{x}(k)| z(k) \dot{x}(k)|z(k)||\dot{x}(k)||z(k)| z(k) \dot{x}(k)|z(k)|^{2}\right\}
\end{aligned}
$$

Prior to installation, the parametric variables, $\theta_{i}$, are determined by using standard model fitting techniques applied to experimental data collected from the MR damper [24]. It should be noted that the parameters, $\theta_{i}$, are specified as functions of the damper command voltage:

$$
\begin{gathered}
F(k)=(0.0083 V+0.005) \dot{x}(k)+z(k) \\
z(k)=z(k-1)+\Delta t\left[\Phi(k-1)^{\mathrm{T}} \boldsymbol{\Theta}\right] \\
\theta_{1}(V)=-13.3 V^{3}+23.0 V^{2}+1.0 V+1.1 \\
\theta_{2}(V)=-161.6 V^{2}-88.7 V-389.3 \\
\theta_{3}(V)=-5.0 V^{2}-169.2 V-160.4 \\
\theta_{4}(V)=-0.64 V^{2}-8.03 V-0.78 \\
\theta_{5}(V)=0.35 V^{2}-6.8 V-0.32
\end{gathered}
$$

The parametric model is tuned using the units of $\mathrm{m} / \mathrm{s}$ for the damper shaft velocity and $\mathrm{MN}$ for the MR damper force.

\subsection{Sensor instrumentation of the test structure}

Two redundant data acquisition systems are installed within the steel structure to conduct realtime feedback control during seismic excitation. The first data acquisition system is entirely wireless and is assembled from a network of wireless sensor prototypes. The second system consists of a tethered data acquisition system permanently installed for testing and controlling specimens on the NCREE facility shaking table. The role of the second system is to serve as a baseline to which the performance of the wireless control system will be compared.

As shown in Figure 2(a), the wireless control system consists of one wireless sensor installed upon each level of the structure (four wireless sensors in total). The wireless sensors installed on the first, second, and third floors are denoted as S1, S2, and S3, respectively. These wireless sensors are responsible for measuring the lateral response of each floor using two sensing transducers interfaced. The first transducer is the Tokyo Sokushin VSE-15-AM servo velocity 
meter. The VSE-15 is capable of measuring velocities within a $0.1-70 \mathrm{~Hz}$ range and up to a maximum magnitude of $1 \mathrm{~m} / \mathrm{s}$. The sensitivity constant of the sensor is $10 \mathrm{~V} /(\mathrm{m} / \mathrm{s})$ and outputs its measurement upon a -10 to $10 \mathrm{~V}$ output signal. To interface to the wireless sensors, a signal conditioning circuit is designed to shift the zero mean sensor output to $2.5 \mathrm{~V}$ and to de-amplify the output by a factor of 4 . The second transducer installed on each floor is the Crossbow CXL02 microelectromechanical system (MEMS) accelerometer. This low-cost accelerometer can measure accelerations from 0 to $50 \mathrm{~Hz}$ with a maximum magnitude of $19.6 \mathrm{~m} / \mathrm{s}^{2}$. The accelerometer, with a sensitivity of $0.102 \mathrm{~V} /\left(\mathrm{m} / \mathrm{s}^{2}\right)$, has a voltage output range from 0 to $5 \mathrm{~V}$.

The fourth wireless sensor, denoted as $\mathrm{C} 1$, is mounted to the surface of the shaking table in the vicinity of the MR damper. This wireless sensor is responsible for measuring the base excitation, determining control forces, and issuing command signals to the MR damper. To measure the base excitation, a Tokyo Sokushin VSE-15 velocity meter and Crossbow CXL02 accelerometer are interfaced. The actuation channel of wireless sensor $\mathrm{C} 1$ is connected to a dSPACE real-time input/output board that controls the operation of the MR damper power supply. To initiate the operation of the wireless control system and to log data wirelessly transmitted by the wireless sensors during testing, a laptop computer is installed in the lab roughly $100 \mathrm{~m}$ from the test structure. A $2.4 \mathrm{GHz}$ XStream wireless radio is interfaced to the laptop computer for recording the flow of data in the network of wireless sensors.

The resolution of the tethered data acquisition system is 16 bits and offers a total of 128 sensor channels. To obtain an accurate measurement of the state of the system during excitation, both velocity meters and linear variable displacement transducers (LVDT) are installed at each floor to measure the absolute velocity and displacement of the structure. A second set of Tokyo Sokushin VSE-15-AM velocity meters are installed adjacent to the velocity meters interfaced to the wireless monitoring system. To measure the absolute displacement of each degree of freedom of the structure, Temposonics II position sensors with a range of $400 \mathrm{~mm}$ and a displacement resolution of $25 \mu \mathrm{m}$ are installed between each floor and a static reference frame constructed to the side of the shaking table. To measure the damper response, a Temposonics II position sensor is also mounted to the damper to measure shaft displacements while a $50 \mathrm{kN}$ load cell is installed between the damper and the steel bracing system.

\section{WIRELESS CONTROL SYSTEM ARCHITECTURE}

A centralized architecture is proposed for the wireless control system with wireless sensor $\mathrm{C} 1$ placed at the centre of the system. Wireless sensor $\mathrm{C} 1$ is responsible for the collection of structural response data (velocity or acceleration) from wireless sensors S1, S2, and S3. Wireless sensors S1, S2, and S3 are responsible only for the measurement of the structural response when queried by wireless sensor $\mathrm{C} 1$. Upon receipt of response data, wireless sensor $\mathrm{C} 1$ is also given the responsibility to determine a control force to be applied to the structure using the MR damper. After calculating the optimal control force, the wireless sensor then issues a command signal to the MR damper.

Two centralized control solutions will be embedded in wireless sensor $\mathrm{C} 1$ depending upon the sensing transducer interfaced to the wireless control system. When servo velocity meters are used, a velocity feedback control solution is employed. In contrast, if accelerometers are used in lieu of velocity meters, then a steady-state Kalman filter is implemented for estimation of the 
state of the structure for full-state feedback control. In both control solutions, LQR is employed. The LQR control solution is optimal since it minimizes a desired response parameter, $\mathbf{y}(t)$, by exerting a minimal amount of control effort [27].

\subsection{Centralized linear quadratic regulation control}

The equation of motion of a multiple degree-of-freedom structure, defined by $n$ degrees of freedom, is formulated in state-space form:

$$
\begin{gathered}
\dot{\mathbf{z}}(t)=\mathbf{A z}(t)+\mathbf{B u}(t)+\mathbf{H f}(t) \\
\mathbf{z}(t)=\left\{\begin{array}{c}
\mathbf{x}(t) \\
\dot{\mathbf{x}}(t)
\end{array}\right\}, \quad \mathbf{A}=\left[\begin{array}{cc}
0 & \mathbf{I} \\
-\mathbf{M}^{-1} \mathbf{K} & -\mathbf{M}^{-1} \mathbf{C}_{\text {damp }}
\end{array}\right]
\end{gathered}
$$

The state response of the structure, $\mathbf{z}$, due to applied external loading, $\mathbf{f}$, is a vector containing the lateral displacement and velocity of each floor relative to the structure base. The properties of the structure, namely mass, $\mathbf{M}$, stiffness, $\mathbf{K}$, and damping, $\mathbf{C}_{\mathrm{damp}}$, are used to derive the system matrix, A. If the structure is controlled, the control forces, $\mathbf{u}$, applied by the control system are included in the state-space equation of motion. The locations of the external system loading, $\mathbf{f}$, and the internal control forces, $\mathbf{u}$, are established by the location matrices $\mathbf{H}$ and $\mathbf{B}$, respectively. The structural response parameter to be minimized by the LQR control solution is written as a linear function of the state response:

$$
\mathbf{y}(t)=\mathbf{C z}(t)
$$

The LQR control solution derives an optimal state trajectory of the state response, $\mathbf{z}$, by simultaneously minimizing the response parameter, $\mathbf{y}$, and the control effort, $\mathbf{u}$. The optimal state trajectory is determined by minimization of a scalar cost function, $J$, over the system time trajectory:

$$
J=\int_{0}^{t} \mathbf{y}^{\mathrm{T}} \mathbf{y}+\mathbf{u}^{\mathrm{T}} \mathbf{R} \mathbf{u}
$$

A weighting matrix, $\mathbf{R}$, is included in the scalar cost function so that the importance of minimizing the control effort relative to minimizing the structural response parameter can be explicitly expressed. Minimization of the scalar cost function, $J$, by Lagrangian methods results in a constant gain matrix, $\mathbf{G}$, that when multiplied by the state of system, $\mathbf{z}$, provides the optimal control forces: $\mathbf{u}(t)=\mathbf{G z}(t)$.

For the three-storey steel structure, the system is modelled as a lumped mass shear structure. The structural response is defined by the deflection of each floor relative to the base of the structure. The lumped mass matrix and the stiffness matrix are formulated for the three-storey steel structure assuming each floor has a mass of $6000 \mathrm{~kg}$ and an inter-storey stiffness of $2.1 \times 10^{6} \mathrm{~N} / \mathrm{m}$. The damping matrix of the structure is derived by the Rayleigh damping method with the damping ratios of the first two modes assumed to be $3 \%$ of critical damping [28]. The mass, stiffness and damping matrices $\left(\mathbf{M}, \mathbf{K}\right.$, and $\left.\mathbf{C}_{\mathrm{damp}}\right)$ are used to calculate the system matrix, $\mathbf{A} \in \mathfrak{R}^{6 \times 6}$. With the MR damper installed at the base of the structure, the actuator location matrix, $\mathbf{B} \in \mathfrak{R}^{6 \times 1}$, is formulated as $\mathbf{B}=\left[\begin{array}{lllllll}0 & 0 & 0 & 1 / 6000 & 0 & 0\end{array}\right]^{\mathrm{T}}$. Similarly, the location matrix of the applied seismic lateral force, $\mathbf{H} \in \mathfrak{R}^{6 \times 1}$, is derived as $\mathbf{H}=\left[\begin{array}{llllll}0 & 0 & 0 & -1 & -1 & -1\end{array}\right]^{\mathrm{T}}$ with $\mathbf{f}(t)$ equal to the base acceleration. 
Two LQR control solutions are formulated to minimize two different response parameters. First, the displacement and velocity response of each floor relative to the base of the structure is minimized:

$$
\mathbf{C}_{1}=\left[\begin{array}{cc}
I & 0 \\
0 & 10 I
\end{array}\right]_{6 \times 6}
$$

The LQR control gain, $\mathbf{G}_{1} \in \mathfrak{R}^{1 \times 6}$, that minimizes the relative displacement and velocity of each floor is found by minimization of the cost function:

$$
J=\int_{0}^{t_{f}} \mathbf{z}^{\mathrm{T}} \mathbf{C}_{1}^{\mathrm{T}} \mathbf{C}_{1} \mathbf{z}+\mathbf{u}^{\mathrm{T}} \mathbf{R} \mathbf{u}
$$

where $\mathbf{C}_{1}^{\mathrm{T}} \mathbf{C}_{1}$ and $\mathbf{R}$ are both positive definite matrices that ensure the cost function can be minimized. A second LQR gain matrix, $\mathbf{G}_{2}$, is formulated to minimize the inter-storey drift of the structure by selection of the following $\mathbf{C}$ matrix:

$$
\mathbf{C}_{2}=\left[\begin{array}{cccccc}
1 & 0 & 0 & 0 & 0 & 0 \\
-1 & 1 & 0 & 0 & 0 & 0 \\
0 & -1 & 1 & 0 & 0 & 0
\end{array}\right]
$$

The LQR control solutions, as presented above, are formulated in the continuous-time domain. However, the wireless control system will operate in the discrete-time domain by commanding the MR damper on a fixed time interval, $\Delta t$, where $t=k \Delta t$ :

$$
u_{\mathrm{MR}}(k)=\mathbf{G}_{d} \mathbf{z}(k)
$$

The equivalent gain matrices in the discrete-time domain, $\mathbf{G}_{d 1}$ and $\mathbf{G}_{d 2}$, are determined by discretizing the continuous-time system using the sample time step and ZOH approximations.

\subsection{Communication scheme for wireless control system}

To maximize the effectiveness of the control system, the smallest possible discrete time step, $\Delta t$, is desired. For the wireless control system, the sample time will depend upon the time needed for the reliable exchange of data between the wireless sensors. Since all of the wireless sensors share the $2.4 \mathrm{GHz}$ wireless channel, a reliable medium access control (MAC) scheme must be implemented to ensure no two sensors contend for the bandwidth at the same time. Contention for the limited bandwidth would adversely affect the reliability of the wireless communications resulting in data loss.

In the wireless control system, wireless sensor $\mathrm{C} 1$ is designed to be the centralized coordinator of the entire system. At each time step, wireless sensor $\mathrm{C} 1$ broadcasts a wireless beacon signal asking each sensor to report its measurement data. Upon receipt of the beacon signal, wireless sensor S1 sends its sensor data to wireless sensor C1. Simultaneously, wireless sensors S2 and S3 remain idle for a period of time to allow the exchange of data between $\mathrm{S} 1$ and $\mathrm{C} 1$ to complete. After a short time period, wireless sensor $\mathrm{S} 2$ establishes communication with $\mathrm{C} 1$ to transfer its state data. Shortly after this transfer, wireless sensor S3 communicates its state data to $\mathrm{C} 1$. This approach effectively divides the sample time window into small segments with each communication step provided exclusive access to the wireless medium. As shown in Figure 3, 


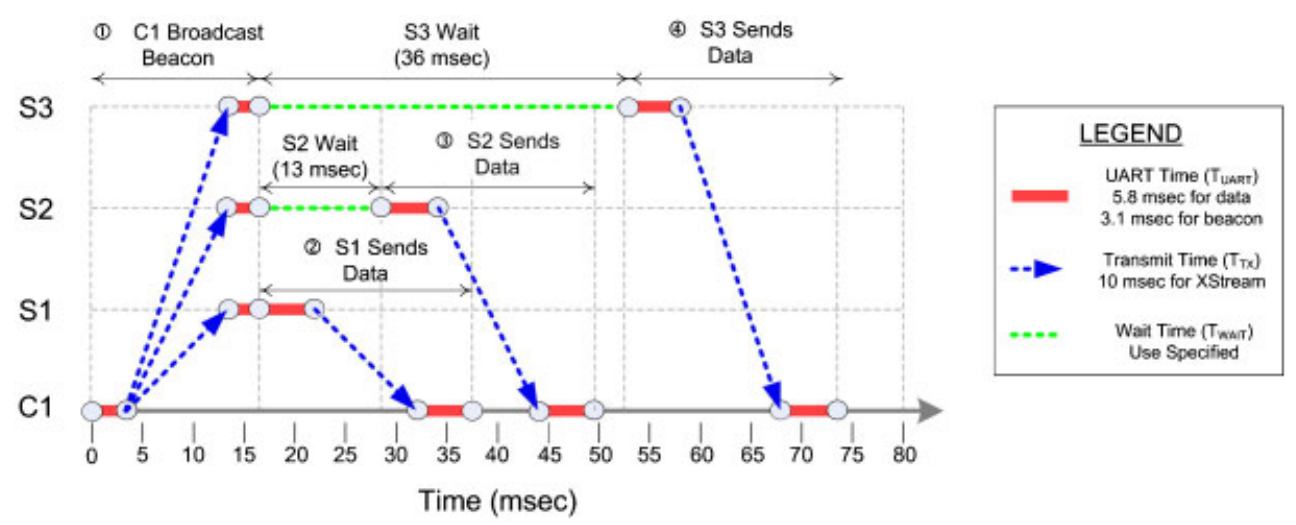

Figure 3. Timing diagram detailing the medium access control scheme of the wireless control system.

this TDMA MAC scheme ensures no two radios attempt to communicate their data to wireless sensor $\mathrm{C} 1$ at the same time.

Prior to installation in the test structure, the timing of the TDMA MAC scheme is optimized to be as fast as possible. In optimizing the MAC scheme for the Maxstream 24XStream radio, the time needed for the exchange of data between two wireless sensors is measured in the laboratory. The peer-to-peer communication time is dependent upon the size of the data packet used to transmit data. For example, the initial beacon signal generated by wireless sensor $\mathrm{C} 1$ is 6 bytes long while the packets transmitting data from sensors S1, S2 and S3 are 11 bytes; as a result, data packets take longer to communicate than beacon packets. The time needed to transfer each wireless packet is broken down to three parts. First, the time needed by a transmitting radio to receive a packet via a serial port from the sensor microcontroller is measured to be 3.1 and $5.8 \mathrm{~ms}$ for the beacon and data packets, respectively. This time is a function of the speed of the universal asynchronous receiver/transmitter (UART) serial port (19200 bits per second). The second part is the time for the modulation of the packet upon the wireless channel; irrespective of the packet size, this time is measured to be $10 \mathrm{~ms}$. The last part is the time needed by the radio to send the wireless packet received to the microcontroller via the serial port. This time is identical to the time it takes to communicate the same packet on the serial port: $3.1 \mathrm{~ms}$ for the beacon packet and $5.8 \mathrm{~ms}$ for the data packet.

The total time needed to complete the transmission of the beacon signal from the microcontroller of $\mathrm{C} 1$ to the microcontroller of S1, S2, and S3 is $16.2 \mathrm{~ms}$. After receipt of the beacon signal, wireless sensor $\mathrm{S} 1$ immediately initiates its communication lasting $21.6 \mathrm{~ms}$ while wireless sensor S2 and S3 back off for 13 and $36 \mathrm{~ms}$, respectively. At precisely $13 \mathrm{~ms}$ after receiving the beacon signal, wireless sensor $\mathrm{S} 2$ wirelessly transmits its state data. Similarly, $36 \mathrm{~ms}$ after receiving the beacon signal, wireless sensor $\mathrm{S} 3$ sends its data to $\mathrm{C} 1$. The entirety of the exchange of data takes $74 \mathrm{~ms}$. Simultaneous to the receipt of state data, wireless sensor $\mathrm{C} 1$ is making the appropriate calculations to determine the control signal to be applied to the MR damper. Including the time needed to determine the optimal control action, the total time step for the wireless control system is set to $\Delta t=0.08 \mathrm{~s}(12.5 \mathrm{~Hz})$. Admittedly, this is a slow sample rate that suggests this specific wireless radio (24XStream) is not very scalable for real-time control beyond a small number of wireless nodes (such as 3 or 4 nodes). 
Table I. Pseudo-code detailing the computational elements of the wireless sensor $\mathrm{C} 1$ during feedback control.

\begin{tabular}{|c|c|c|}
\hline Computing task & Component & Description \\
\hline \multicolumn{3}{|l|}{ for $k=1: N$} \\
\hline BeaconSystem & Wireless channel & Beacons system at start of time step \\
\hline RecordBaseResp & Sensing interface & Records base response, $v_{0}$ or $a_{0}$ \\
\hline WirelessReceive(1) & Wireless channel & Receive from floor $1, v_{1}$ or $a_{1}$ \\
\hline RelativeResp & Computing core & Find relative response (e.g. $v_{1}-v_{0}$ ) \\
\hline WirelessReceive(2) & Wireless channel & Receive from floor $2, v_{2}$ or $a_{2}$ \\
\hline RelativeResp & Computing core & Find relative response (e.g. $\left.v_{2}-v_{0}\right)$ \\
\hline WirelessReceive(3) & Wireless channel & Receive from floor $3, v_{3}$ or $a_{3}$ \\
\hline RelativeResp & Computing core & Find relative response (e.g. $\left.v_{3}-v_{0}\right)$ \\
\hline \multicolumn{3}{|l|}{ if (acceleration==True) } \\
\hline KalmanFilter & Computing core & Estimate full state, $\mathbf{z}$, from $\mathbf{y}$ (Equation (13)) \\
\hline else & Comnuting core & Assemble the partial state $\mathrm{z}=\left\{0 \dot{\mathbf{x}}^{\mathrm{T}}\right\}^{\mathrm{T}}$ \\
\hline end & Computing core & \\
\hline CalculateLQRForce & Computing core & LQR control law to find $u_{\mathrm{MR}}(k+1)$ (Equation (11)) \\
\hline GenerateVoltParamTable & Computing core & For discrete voltage levels, find $\theta_{i}$ (Equation (4)) \\
\hline GenerateForceTable & Computing core & $\begin{array}{l}\text { For discrete voltage levels, find } F(k+1) \text { (Equation } \\
\text { (4)) }\end{array}$ \\
\hline CompareForces & Computing core & Compare $u_{\mathrm{MR}}(k+1)$ to possible set $F(k+1)$ \\
\hline CommandMRDamper & Actuation interface & Apply voltage attaining $F(k+1)$ \\
\hline UpdateBoucWen & Computing core & $\begin{array}{l}\text { Update the Bouc-Wen hysteretic model (Equation } \\
\text { (4)) }\end{array}$ \\
\hline $\begin{array}{l}\text { Wait } \\
\text { end }\end{array}$ & Computing core & Wait a while to achieve a precise time step of $0.08 \mathrm{~s}$ \\
\hline
\end{tabular}

\subsection{Embedded software for the wireless controller}

Embedded software in the wireless controller $(\mathrm{C} 1)$ automates the activities of the entire wireless control system. The software is written in a modular fashion in the high-level $\mathrm{C}$ programming language. Before downloading to the wireless sensor flash memory, the compiler converts it to machine language. Table I provides an overview of the embedded software which is written as a single-threaded algorithm. The unique aspects of the implementation are described in greater detail below.

The major computational module of the embedded software is the calculation of the optimal control force using response data measured by the wireless control system. When velocity sensors are interfaced to the wireless sensors, half of the state response of the system is measured. For example, the velocity meter installed on the $i$ th floor provides a measurement of the absolute velocity, $v_{i}$, allowing the relative velocity components of the state to be determined: $\dot{\mathbf{x}}(k)=\left\{\begin{array}{lll}v_{1}-v_{0} & v_{2}-v_{0} & v_{3}-v_{0}\end{array}\right\}^{\mathrm{T}}$. To simplify the calculation of the LQR control force, the displacement components of the state are ignored: $\mathbf{z}(k)=\left\{\begin{array}{llll}0 & 0 & 0 & \dot{\mathbf{x}}(k)^{\mathrm{T}}\end{array}\right\}^{\mathrm{T}}$. Using the truncated state, the desired control force to be applied by the MR damper, $u_{\mathrm{MR}}(k)$, is calculated by Equation (11) using the LQR gain matrices $\left(\mathbf{G}_{d 1}\right.$ or $\left.\mathbf{G}_{d 2}\right)$. Execution of this equation by the wireless sensor consists only of the multiplication and addition of floating point numbers and 
takes fractions of a millisecond to complete. Should a velocity reading not be received by wireless sensor $\mathrm{C} 1$ within the allotted time window of the TDMA communication scheme, the absolute velocity of that floor is designated as zero for that time step $\left(v_{i}=0\right)$.

In practice, structural control systems employ accelerometers to measure the dynamic structural response. However, feedback of accelerations require Kalman estimation at the controller. If the equation describing the dynamic equilibrium of the structure is written in its discretized form

$$
\begin{gathered}
\mathbf{z}(k+1)=\mathbf{\Phi z}(k)+\mathbf{\Gamma} u_{\mathrm{MR}}(k) \\
\mathbf{y}(k)=\left\{\begin{array}{lll}
\ddot{x}_{1} & \ddot{x}_{2} & \ddot{x}_{3}
\end{array}\right\}^{\mathrm{T}}=\left[\begin{array}{l}
-\mathbf{M}^{-1} \mathbf{K}-\mathbf{M}^{-1} \mathbf{C}_{\mathrm{damp}}
\end{array}\right] \mathbf{z}(k)+[\mathbf{B}] u_{\mathrm{MR}}(k)=\mathbf{C z}(k)+\mathbf{D} u_{\mathrm{MR}}(k)
\end{gathered}
$$

then the measured state response, $\mathbf{y}(k)$, can be stated as the vector of accelerations of each degree of freedom of the structure, $\ddot{x}_{i}$, relative to the base. The matrices $\boldsymbol{\Phi}$ and $\boldsymbol{\Gamma}$ are the discretetime equivalents to the system, A, and location, $\mathbf{B}$, matrices, respectively. The steady-state Kalman estimator is determined so that the full state at step $k+1$ can be estimated:

$$
\hat{\mathbf{z}}(k+1)=(\boldsymbol{\Phi}-\mathbf{L C}+\boldsymbol{\Gamma} \mathbf{G}-\mathbf{L D G}) \hat{\mathbf{z}}(k)+\mathbf{L y}(k)
$$

where the estimated state, $\hat{\mathbf{z}}$, is determined based on the steady-state Kalman gain matrix, $\mathbf{L}$. The steady-state Kalman estimator is encoded in the wireless sensor so that an estimate for the full state can be made at each time step using measured accelerations. The wireless sensor then uses the estimated state to calculate the desired control force to be applied by the MR damper: $u_{\mathrm{MR}}(k)=\mathbf{G}_{d} \hat{\mathbf{z}}(k)$. Again, should a data point not be received by the wireless sensor, the measured state response, $\mathbf{y}(k)$, would have a zero in lieu of the true measured value not received.

After wireless sensor $\mathrm{C} 1$ calculates the control force, $u_{\mathrm{MR}}$, it must then determine the appropriate command voltage that will generate the reaction force in the damper. The linearly parameterized Bouc-Wen model (Equation (4)) derived for the $20 \mathrm{kN}$ MR damper is coded as the last embedded software module. Prior to installation in the test structure, the Bouc-Wen model parameters, $\theta_{i}$, are calculated for 11 voltage values ranging from 0 to $1 \mathrm{~V}$ (in $0.1 \mathrm{~V}$ increments). A table of the five model parameters are stored for the 11 voltage levels. At each time step, the relative velocity of the first storey, $\dot{x}_{1}(k)$, and the hysteretic restoring force of the damper, $z(k)$, are used to calculate the MR damper force, $F(k+1)$, corresponding to each voltage level. The 11 values for $F(k+1)$ are then compared to the desired control force, $u_{\mathrm{MR}}(k)$; the force closest to the desired control force is noted and the corresponding voltage applied. The final step is to update the hysteretic restoring force, $z(k+1)$ which is saved by the wireless sensor for the next time step. At the very end of the time step, the embedded microcontroller is paused briefly to ensure the time step is precisely $0.08 \mathrm{~s}(12.5 \mathrm{~Hz})$. The embedded code is executed multiple times for a total number of steps, $N$.

\section{VALIDATION OF THE WIRELESS CONTROL SYSTEM}

To assess the performance of the wireless control system, three earthquake excitations are selected for application to the test structure by the shaking table. Horizontal acceleration timehistory records corresponding to the 1940 El Centro (Imperial Valley Irrigation District Station, North-South), 1999 Chi-Chi (TCU-076 Station, North-South) and 1995 Kobe (JMA Station, 
Table II. Overview of the experimental tests conducted to assess the efficacy of the wireless control system.

\begin{tabular}{lcccccccc}
\hline & \multicolumn{2}{c}{ Passive control } & & \multicolumn{2}{c}{ Velocity feedback } & & \multicolumn{2}{c}{ Acceleration feedback } \\
\cline { 2 - 3 } Earthquake record & $0 \mathrm{~V}$ & $1 \mathrm{~V}$ & & $\mathbf{G}_{d 1}$ & $\mathbf{G}_{d 2}$ & & $\mathbf{G}_{d 1}$ & $\mathbf{G}_{d 2}$ \\
\hline El Centro NS (Imperial) & Test 1 & Test 2 & & Test 7 & Test 10 & & Test 11 & Test 14 \\
Chi-Chi NS (TCU-076) & Test 3 & Test 4 & & Test 8 & - & & Test 12 & - \\
Kobe NS (JMA) & Test 5 & Test 6 & & Test 9 & - & & Test 13 & - \\
\hline
\end{tabular}

North-South) earthquakes are selected. The El Centro ground motion record is a far-field record whereas the Chi-Chi and Kobe records are near-field records. The absolute peak acceleration recorded for these three earthquakes are $3.42,4.20$, and $8.18 \mathrm{~m} / \mathrm{s}^{2}$, respectively. However, to keep the test structure in its linear elastic regime, the peak absolute accelerations of the El Centro, Chi-Chi and Kobe ground motion records are scaled to 1, 0.9, and $0.85 \mathrm{~m} / \mathrm{s}^{2}$, respectively.

Combined with the different sensing transducers utilized by the wireless monitoring system (velocity meters versus accelerometers) and the different gain matrices derived $\left(\mathbf{G}_{d 1}\right.$ and $\left.\mathbf{G}_{d 2}\right)$, a total of eight unique tests are conducted to assess the performance of the wireless control system. In addition, the same ground excitations are applied to the structure with the damper fixed to its minimum and maximum damping coefficients ( 0 and $1 \mathrm{~V}$, respectively). The response of the structure using the wireless feedback control system will be compared to the damper configured in its passive setting. Table II summarizes the 14 tests that are used to assess the performance of the wireless control system.

In addition to the tests conducted using the wireless control system, the tethered laboratory data acquisition system is also used to separately perform real-time feedback control of the test structure. Again, the structure is controlled by the tethered system using both velocity meters and accelerometers. Since communication in the tethered system is performed using coaxial wiring, the tethered control system can operate using a much higher sample rate. The tethered monitoring system employs a sample rate of $200 \mathrm{~Hz}$ which is an order of magnitude faster than the $12.5 \mathrm{~Hz}$ sample rate of the wireless control system.

For all of the closed-loop control tests performed, two evaluation criteria will be considered. First, the absolute maximum inter-storey drift will be calculated for each floor of the structure. The second metric for assessing the effectiveness of the closed-loop control system will be a scalar cost function looking at the total kinetic and strain energy experienced by the structure over the duration of the base excitation:

$$
J=\sum_{k} \mathbf{z}(k)^{\mathrm{T}}\left[\begin{array}{cc}
\mathbf{K} & 0 \\
0 & \mathbf{M}
\end{array}\right] \mathbf{z}(k)
$$

The cost function for each closed-loop control test, $J_{\mathrm{C}}$, will be compared to that of the structure controlled in a passive configuration with the MR damper set to its smallest damping coefficient ( $0 \mathrm{~V}$ configuration), $J_{0}$ :

$$
\bar{J}=J_{\mathrm{C}} / J_{0}
$$

A cost-function ratio $\bar{J}<1$ indicates the closed-loop control system outperforms the passive damper set to its minimum damping coefficient. A number greater than 1 would indicate the control system is working to the detriment of the structure. 

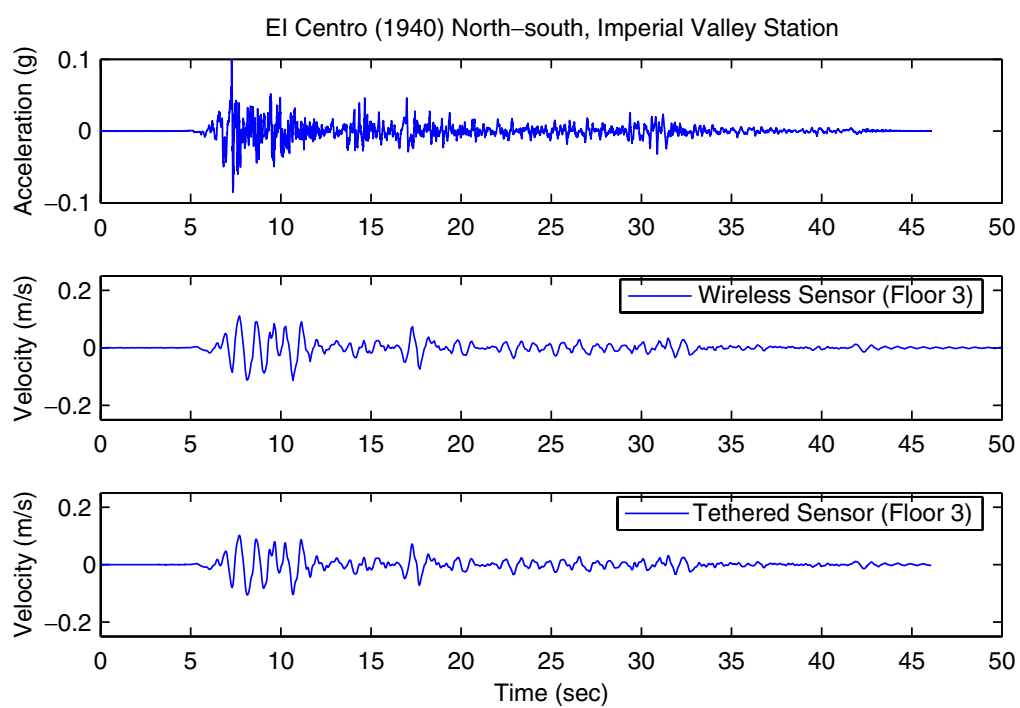

Figure 4. Velocity response of the 3rd storey of the test structure under the El Centro NS ground motion: (top) applied ground acceleration, and the velocity measured by the (middle) wireless and (bottom) tethered sensors.

\subsection{Velocity feedback control}

In total, four tests are conducted to assess the performance of the wireless control system using a velocity feedback solution. Three of the tests, Tests 7 (El Centro), 8 (Chi-Chi) and 9 (Kobe), adopt the first gain matrix, $\mathbf{G}_{d 1}$, while Test 10 (El Centro) adopts the second gain matrix, $\mathbf{G}_{d 2}$. Recall, $\mathbf{G}_{d 1}$ is designed to minimize (regulate) the displacement and velocity of each floor relative to the base; similarly, $\mathbf{G}_{d 2}$ is designed to minimize inter-storey drifts. Consider Test 7 with the El Centro NS ground motion applied to the structure while the wireless control system mitigates the structural response. Figure 4 presents the corresponding velocity response of the structure at the top-most storey (3rd floor). As can be seen, the response measured by the velocity meter interfaced to the wireless sensor S3 is identical to that recorded by the laboratory data acquisition system using a separate velocity meter. The time history records recorded at other degrees of freedom also reveal the response measured by wireless sensors (S2 and S1) are identical to those recorded by the tethered data acquisition system. It should be noted that the greatest response in the structure occurs from roughly $5-15 \mathrm{~s}$ during which time the ground motion attains its peak absolute value $(0.1 \mathrm{~g}$ at $7.28 \mathrm{~s})$. The displacement response of the controlled structure during Test 7 , as measured by the laboratory tethered data acquisition system, is presented in Figure 5. The displacement response of the controlled structure using the wireless structural control system is superimposed upon the displacement response recorded when the MR damper is set to its minimum $(0 \mathrm{~V})$ and maximum $(1 \mathrm{~V})$ damping coefficients. The response of the structure during the interval of greatest response $(5-15 \mathrm{~s})$ is clearly reduced by the velocity feedback wireless control system compared to when the damper is fixed at the minimum and maximum damping coefficients. Similar observations can be made for all of the structure's three stories. 

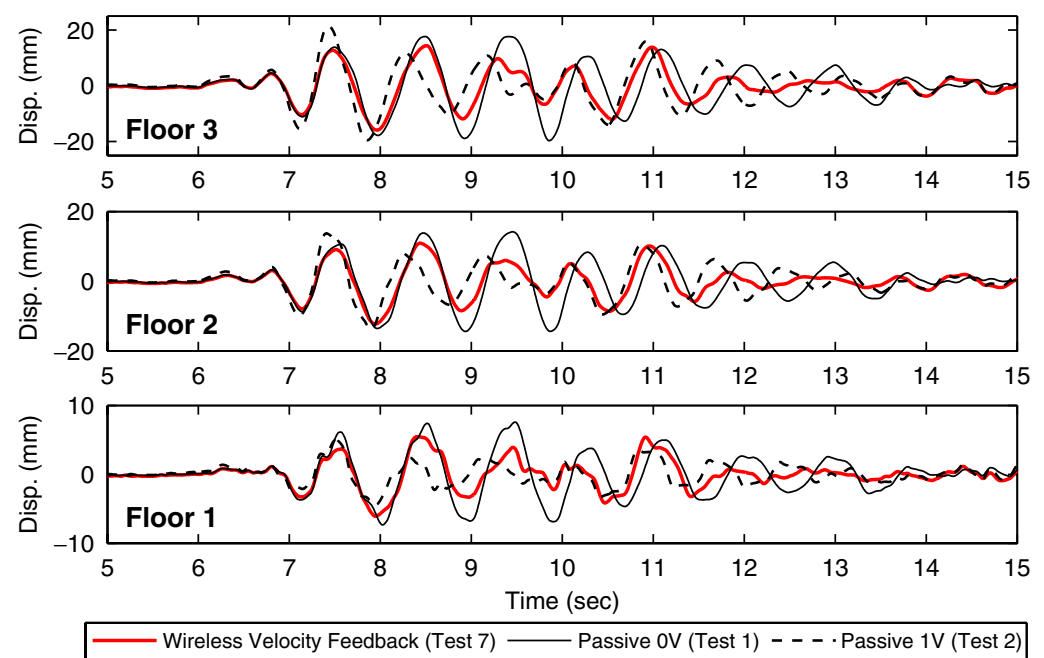

Figure 5. Segment of structural displacement response time histories when controlled (wireless velocity feedback and minimum and maximum passive damping) during El Centro NS ground motion: (top) floor 3 ; (middle) floor 2; and (bottom) floor 1.

At each time step, wireless sensor $\mathrm{C} 1$ uses the measured velocity of each floor to calculate the LQR control force (Equation (11)). The modified Bouc-Wen model is then used to determine the command voltage to be applied to the MR damper by the wireless sensor. Using the load cell installed in series with the MR damper (see Figure 2), the true force applied by the MR damper is measured by the laboratory tethered data acquisition system. As presented in Figure 6, the measured MR damper reaction force and the desired control force are in strong agreement during Test 7. This agreement suggests the modified Bouc-Wen model is sufficiently accurate for determining command voltages of the MR damper to attain a desired control force in the damper. Also presented in Figure 6 is the command voltage time history issued by wireless sensor $\mathrm{C} 1$ during the experiment.

Plots corresponding to the maximum absolute inter-storey drift and peak relative acceleration of each degree of freedom of the test structure are presented in Figure 7. The performance of the wireless control system is compared to the same response parameters attained when the MR damper is set to minimum and maximum damping coefficients. In addition, the maximum absolute inter-storey drift attained when using an LQR velocity feedback control solution implemented using the laboratory data acquisition system are superimposed upon the plots of Figure 7. It should be noted that the LQR gain matrix used with the tethered control system is derived for the higher sample rate $(200 \mathrm{~Hz})$ using the same response criteria $\left(\mathbf{C}_{1}\right.$ and $\left.\mathbf{C}_{2}\right)$ as the wireless control system. As can be seen for Test 7 (El Centro), the wireless and tethered control system are both effective in reducing the inter-storey drift of the 2 nd and 3 rd storeys of the structure. Furthermore, both control systems outperform the cases when the MR damper is set to a passive state. However, the performance of the wireless control system is not as impressive when the two near field earthquakes (Chi-Chi and Kobe ground motions) are applied to the structure. For both Test 8 (Chi-Chi NS) and 9 (Kobe NS), the tethered monitoring system is effective in reducing the drift response of the structure while the response of the wireless control 

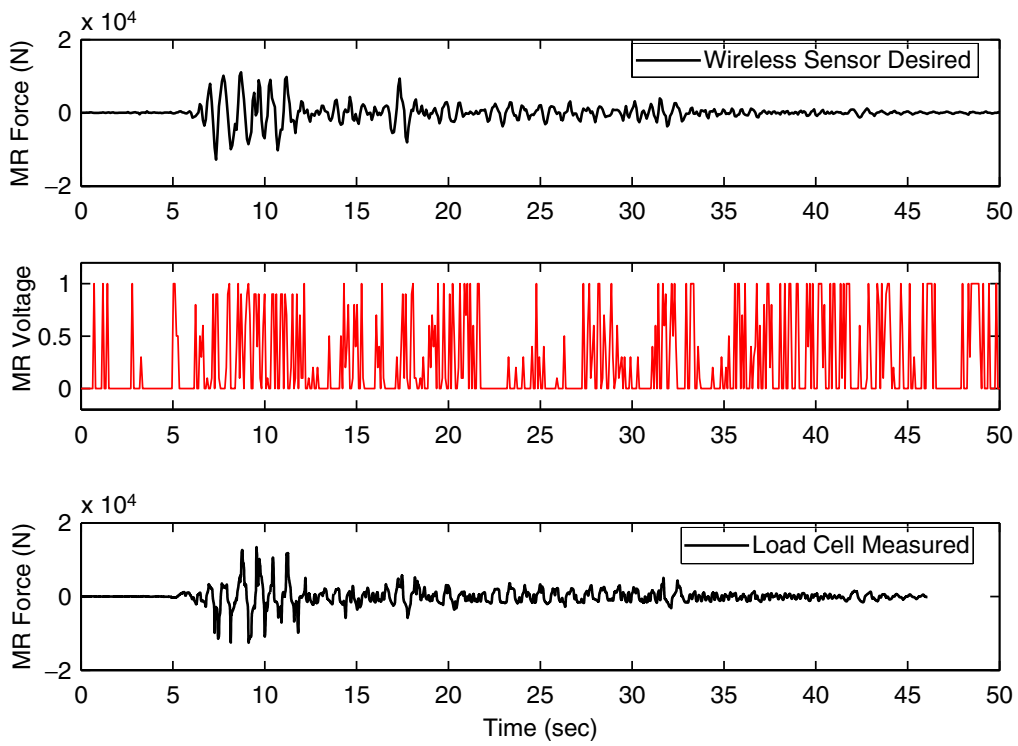

Figure 6. MR damper control force: (top) desired control force calculated; (middle) the voltage command signal issued to the MR damper by the wireless sensor; and (bottom) actual control force measured by the load cell.
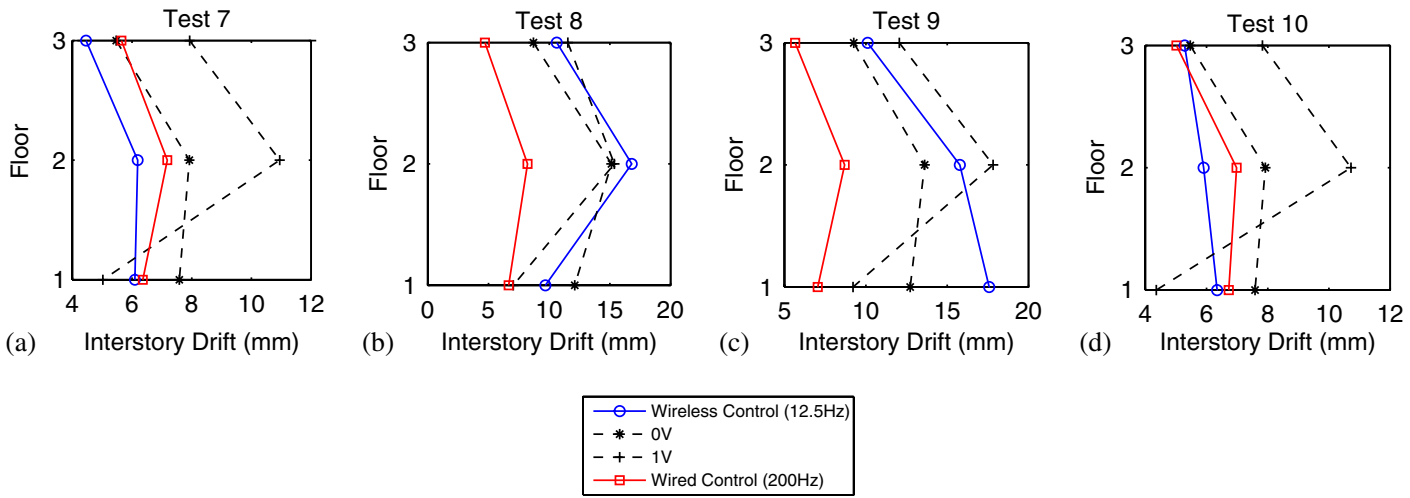

Figure 7. Maximum absolute inter-storey drifts for velocity feedback control using gain $\mathbf{G}_{d 1}$ : (a) El Centro (Test 7); (b) Chi-Chi (Test 8); and (c) Kobe (Test 9) ground motions. When using gain $\mathbf{G}_{d 2}$ :

(d) El Centro (Test 10).

system can be worse than the passive MR tests. The wireless control system's low sample rate and state truncation are likely causes for its poor performance during two near-field excitations. The wired control system, operating at $200 \mathrm{~Hz}$, was able to effectively reduce the structural response using the same response parameters in the LQR formulation $\left(\mathbf{C}_{1}\right)$. For Test 10 , the LQR gain matrix corresponding to the minimization of the inter-storey drift, $\mathbf{G}_{d 2}$, is employed. Again, as presented in Figure 7(d), the wireless control system is effective in reducing the drift 
Table III. Performance assessment using the cost function ratio, $\bar{J}$.

\begin{tabular}{|c|c|c|c|c|c|c|}
\hline \multirow[b]{2}{*}{ Earthquake record } & \multicolumn{2}{|c|}{ Passive control } & \multicolumn{2}{|c|}{ Velocity feedback } & \multicolumn{2}{|c|}{ Acceleration feedback } \\
\hline & $0 \mathrm{~V}$ & $1 \mathrm{~V}$ & $\mathbf{G}_{d 1}$ & $\mathbf{G}_{d 2}$ & $\mathbf{G}_{d 1}$ & $\mathbf{G}_{d 2}$ \\
\hline El Centro NS (Imperial) & 1 & 0.70 & 0.59 & 0.33 & 0.58 & 0.43 \\
\hline Chi-Chi NS (TCU-076) & 1 & 1.10 & 0.90 & - & 0.59 & - \\
\hline Kobe NS (JMA) & 1 & 1.40 & 1.07 & - & 0.59 & - \\
\hline
\end{tabular}

response of the structure to levels lower than those when the MR damper is operated in a passive state.

Plots of the maximum absolute drifts reveal the effectiveness of the wireless control system in mitigating peak, or rather worst-case, response parameters. In contrast, the cost-function ratio, $\bar{J}$, is calculated for each control experiment to assess the wireless control system effectiveness over the entire time history of the excitation (Table III). The cost-function ratios for the El Centro excitations reveal the effectiveness of the wireless control system. Roughly speaking, the control system exhibits 59 and $33 \%$ of the total response energy corresponding to the structure when the minimum MR damping coefficient is set. This is in contrast to when the MR damper is set to its maximum damping coefficient where the structure exhibits $70 \%$ of the response energy compared to the minimum MR damping coefficient configuration. Consistent with the observations previously made during the Chi-Chi and Kobe excitations, the cost-function ratio also reveals the wireless control system is only mildly effective in mitigating the response of the structure during the full time history of the excitation.

\subsection{Acceleration feedback control}

Using $\mathbf{G}_{d 1}$ for acceleration feedback control, the structure is exposed to the El Centro, Chi-Chi and Kobe earthquakes (Tests 11, 12 and 13, respectively). As shown in Figure 8, the wireless control system is effective in reducing the inter-storey drift response of the structure under all three applied ground motions; the drift response is below those corresponding to the MR damper in a passive state (minimum and maximum damping coefficients). When comparing the inter-storey drift performance of the wireless control system to the wired control system, the wireless control system offers superior performance. The second control solution, $\mathbf{G}_{d 2}$ (Test 14), allows the wireless control system to exhibit excellent inter-storey drift mitigation performance. As presented in Figure 8(d), the wireless control system is as effective as the wired control system when implementing this control solution. For both control solutions $\left(\mathbf{G}_{d 1}\right.$ and $\left.\mathbf{G}_{d 2}\right)$, the costfunction ratio $\bar{J}$ reveals the wireless control system is effective over the full excitation, resulting in lower response energy than when the MR damper is set to minimum and maximum damping coefficients.

\subsection{Quality of the wireless communication channel}

In total, 47 tests are conducted using the prototype wireless control system. During each of the closed-loop control tests, wireless sensor $\mathrm{C} 1 \mathrm{logs}$ the reception of data from each wireless sensor installed in the upper levels of the test structure. After the completion of each test, the data received by wireless sensor $\mathrm{C} 1$ are sent to a remote data server where it can be stored and analysed. With the performance of the control system dependent upon the reliability of data 

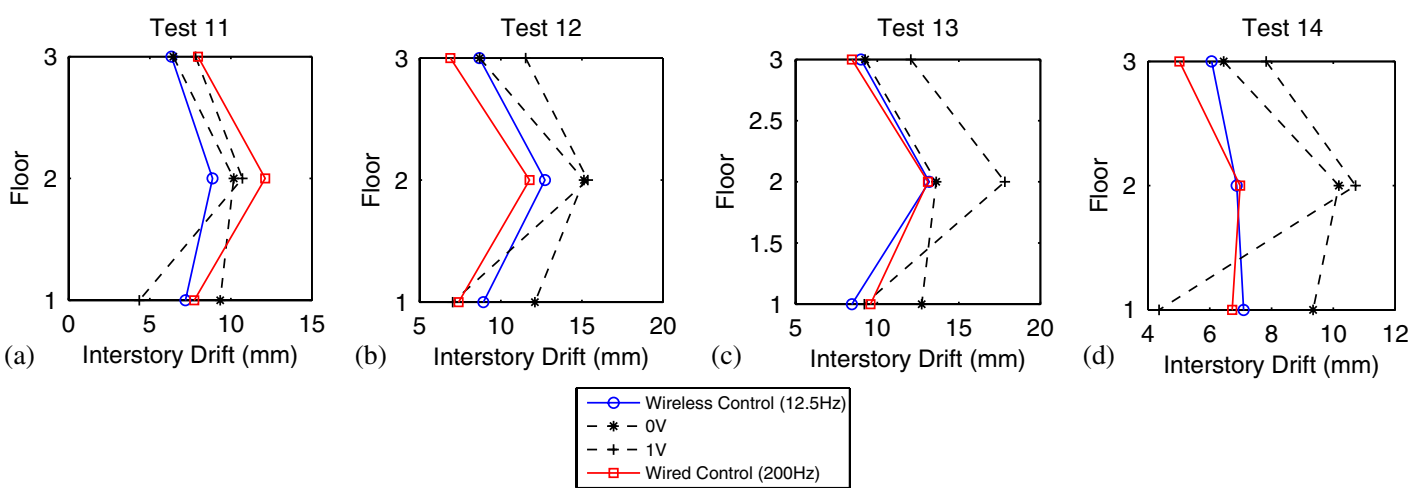

Figure 8. Maximum absolute inter-storey drifts for acceleration feedback control using gain $\mathbf{G}_{d 1}$ : (a) El Centro (Test 11); (b) Chi-Chi (Test 12); and (c) Kobe (Test 13) ground motions. When using gain $\mathbf{G}_{d 2}$ : (d) El Centro (Test 14).
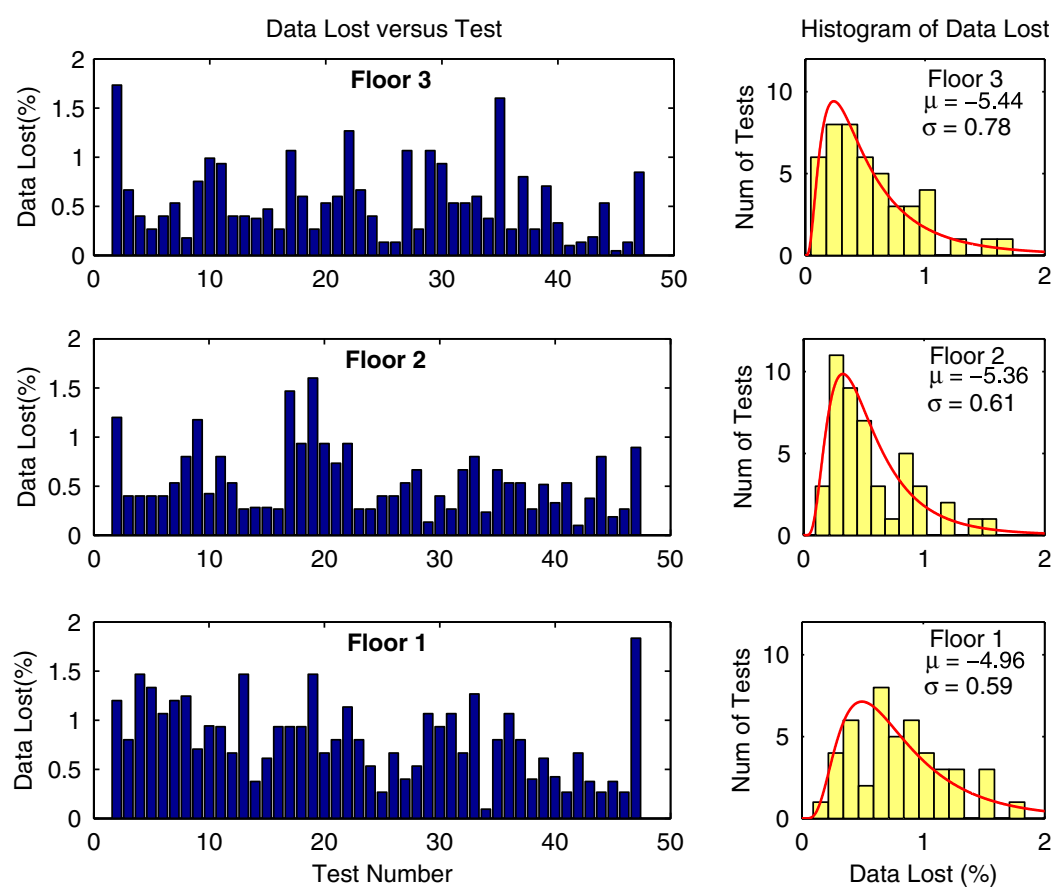

Figure 9. Reliability assessment of the wireless channel: (left column) percentage of lost data points per floor and (right column) histogram of number of tests versus data loss percentage with fitted log-normal distributions.

delivery, the logs of received data by wireless sensor $\mathrm{C} 1$ are analysed to determine how often data are lost by the wireless control system. A bar graph of the percent of data points not received by wireless sensor $\mathrm{C} 1$ is plotted in Figure 9. Only a handful of cases exhibit data losses 
of roughly $2 \%$. However, the vast majority of the tests have data loss rates less than $1 \%$ and in many cases, less than $0.5 \%$. For each floor, a histogram of the percentage of lost data is determined. As seen in Figure 9, the histograms reveal the data loss phenomena in the wireless control system exhibits a log-normal probability distribution function. To illustrate this distribution more clearly, a log-normal distribution is fitted to each histogram and is superimposed. The mean and standard deviations of the fitted log-normal distributions, $\mu$ and $\sigma$, are also presented on each plot. The mean data loss for floors 1, 2, and 3 are determined to be $0.70,0.47$, and $0.43 \%$, respectively.

\subsection{Increasing the sample rate of the wireless control system}

The poor performance of the velocity feedback control solution during near-field seismic ground motions (Tests 8 and 9) is due in part to the slow sample rate employed by the wireless control system. Specifically, the wireless control system is operated at $12.5 \mathrm{~Hz}$ to ensure sufficient time is allotted to each wireless sensors in the TDMA access control communication technique. With most practical structural control systems implemented at high sample [6], future research is needed to improve the speed of wireless control systems defined by high nodal densities. Two possible avenues for future exploration include improved wireless radios offering higher communication speeds and adoption of decentralized control system architectures.

The 24XStream wireless radio suffers from a relatively slow physical protocol layer. In contrast, there exist alternative wireless communication standards offering significantly higher communication speeds. In particular, IEEE802.11 and 802.15.4 wireless standards offer high over-the-air data rates (1000 and $250 \mathrm{kbits} / \mathrm{s}$, respectively). Towards this end, Swartz and Lynch [29] propose the design of a wireless sensor prototype with an IEEE802.15.4 transceiver and actuation interface included. For a three-node wireless control system, they report control system sample rates as high as $40 \mathrm{~Hz}$.

Scaling up wireless control systems to high nodal densities will ultimately saturate the limited bandwidth offered by the wireless communication channel (regardless of the specific radio used). As the wireless control system grows, issues such as data loss in the communication channel and time needed to exchange data between sensors increase faster than at a linear rate. As a result, decentralized control system architectures are attractive candidates for future control systems designed using low-cost wireless sensor networks. In decentralized control architectures, the global system is divided into smaller subsystems in which sensors and actuators are locally controlled. Therefore, the wireless communication ranges are significantly shorter and communication latencies decrease since there are fewer wireless sensors within each subsystem.

\section{CONCLUSIONS}

This study explored the use of wireless sensors within a real-time structural control system. At the core of the proposed wireless control system is a low-cost wireless sensor capable of sensing, actuating, computing, and communicating. A significant portion of the wireless sensor design is the embedded software that automates the operation of the unattended wireless sensor network. To achieve a high level of performance, the embedded software is written to ensure reliable transfer of state data between wireless sensors at each time step. The performance of the wireless control system is validated using a full-scale three-storey steel structure mounted to a shaking 
table. At the base of the structure is a $20 \mathrm{kN}$ MR damper that mitigates the response of the structure during ground motion. The wireless control system proves effective in reducing the inter-storey drifts of each floor during seismic excitation. Particularly for the case of acceleration feedback control, the wireless control system performs at a level of performance equivalent to a baseline wired control system for both far- and near-field seismic excitations. When velocity meters are used in lieu of accelerometers, the wireless control system is effective in reducing the drift response of the structure for far-field ground motions (El Centro) but is unable to outperform the passive damper cases for near-field motions (Chi-Chi and Kobe). This could be the result of the suboptimal nature of the truncated velocity-feedback gain matrix in combination with the low sample rate of the wireless system $(12.5 \mathrm{~Hz})$. During operation, the wireless control system's wireless communication channel proved highly reliable with minimal data loss $(<2 \%)$ occurring during the tests. The general success of the wireless control system presented herein suggests wireless sensor networks are a promising technology capable of operation within a real-time control system.

One limitation encountered in the study was the low sample rate attainable using the wireless sensors. The TDMA approach implemented for the Maxstream 24XStream radios offers a $12.5 \mathrm{~Hz}$ sample rate which is slow for most closed-loop structural control applications. Beyond the four-node system used in this study, larger control systems would encounter even lower sample rates to ensure reliable communication. Future work will explore how larger wireless control systems can be implemented for structural control. Two specific approaches have been proposed including the use of a different wireless radio for communication and adoption of decentralized system architectures.

\section{ACKNOWLEDGEMENTS}

This research is partially funded by the Office of Naval Research (ONR) Young Investigator Program. Additional support was provided by the National Centre for Research on Earthquake Engineering (NCREE) and the National Science Council of Taiwan. Mr Yang Wang is supported by the Stanford Graduate Fellowship at Stanford University. The authors would like to express extreme gratitude to Prof. Kincho H. Law of Stanford University who has provided invaluable expertise in all facets of this project.

\section{REFERENCES}

1. Yao JTP. Concept of structural control. Journal of Structural Division 1972; 98(7):1567-1574.

2. Housner GW, Masri SF, Chaffiakos AG (eds). Proceedings of the First World Conference on Structural Control. Wiley: Los Angeles, CA, 1994.

3. Inoune Y, Seto K, Iemura H, Nishitani A (eds). Proceedings of the Second World Conference on Structural Control. Wiley: Kyoto, Japan, 1998.

4. Casciati F (ed.). Proceedings of the Third World Conference on Structural Control. Wiley: Lake Como, Italy, 2002.

5. Smyth A, Betti R (eds). Proceedings of the Fourth International Workshop on Structural Control. DEStech Publications: New York, NY, 2004.

6. Chu SY, Soong TT, Reinhorn AM. Active, Hybrid, and Semi-active Structural Control: A Design and Implementation Handbook. Wiley: New York, NY, 2005.

7. Casciati F, Magonette G, Marazzi F. Technology of Semiactive Devices and Applications in Vibration Monitoring. Wiley: West Sussex, U.K., 2006.

8. Housner GW, Bergman LA, Caughey TK, Chassiakos AG, Claus RO, Masri SF, Skelton RE, Soong TT, Spencer BF, Yao JTP. Structural control: past, present and future. Journal of Engineering Mechanics 1997; 123(9):897-971.

9. Spencer BF, Nagarajaiah S. State of the art of structural control. Journal of Structural Engineering 2003; 129(7): $845-856$. 
10. Kobori T, Takahashi M, Nasu T, Niwa N, Ogasawara K. Seismic response controlled structure with active variable stiffness system. Earthquake Engineering and Structural Dynamics 1993; 22(11):925-941.

11. Kurata N, Kobori T, Takahashi M, Niwa N, Midorikawa H. Actual seismic response controlled building with semiactive damper system. Earthquake Engineering and Structural Dynamics 1999; 28(11):1427-1447.

12. Gavin HP. Design method for high-force electrorheological dampers. Smart Materials and Structures 1998; 7(5):664-673.

13. Jung HJ, Spencer BF, Ni YQ, Lee IW. State-of-the-art of semiactive control systems using MR fluid dampers in civil engineering applications. Structural Engineering and Mechanics 2004; 17(3-4):493-526.

14. Symans MD, Constantinou MC. Semi-active control systems for seismic protection of structures: a state-of-the-art review. Engineering Structures 1999; 21(6):469-487.

15. Celebi M. Seismic instrumentation of buildings with emphasis on federal buildings. Report 0-7460-68170, United States Geological Survey, Menlo Park, CA, 2002.

16. Lian FL, Moyne J, Tilbury DM. Network design consideration for distributed control systems. IEEE Transactions on Control Systems Technology 2002; 10(2):297-307.

17. Eker J, Cervin A, Horjel A. Distributed wireless control using bluetooth. IFAC Conference on New Technologies for Computer Control, Hong Kong, China, 2001.

18. Nilsson J, Bernhardsson B, Wittenmark B. Stochastic analysis and control of real-time systems with random time delays. Automatica 1998; 34(1):57-64.

19. Ploplys NJ, Kawka PA, Alleyne AG. Closed-loop control over wireless networks. IEEE Control Systems Magazine 2004; 24(3):58-71.

20. Casciati F, Rossi R. Fuzzy chip controllers and wireless links in smart structures. Advances in Smart Technologies in Structural Engineering, Jadwisin, Poland, 2004.

21. Lynch JP. Design of a wireless active sensing unit for localized structural health monitoring. Journal of Structural Control and Health Monitoring 2004; 12(3-4):405-423.

22. Wang Y, Lynch JP, Law KH. Design of a low-power wireless structural monitoring system for collaborative computational algorithms. Proceedings of the SPIE-The International Society for Optical Engineering, San Diego, CA, U.S.A., 2005; 106-117.

23. Lynch JP, Loh K. A summary review of wireless sensors and sensor networks for structural health monitoring. Shock and Vibration Digest 2006; 38(2):91-128.

24. Lin PY, Roschke P, Loh CH. System identification and real application of a smart magnetorheological damper. 2005 IEEE International Symposium on Intelligent Control, Limassol, Cyprus, 2005; 989-994.

25. Smyth AW, Masri SF, Chassiakos AG, Caughey TK. On-line parametric identification of mdof nonlinear hysteretic systems. Journal of Engineering Mechanics 1999; 125(2):133-142.

26. Smyth AW, Masri SF, Kosmatopoulos EB, Chassiakos AG, Caughey TK. Development of adaptive modeling techniques for non-linear hysteretic systems. International Journal of Non-Linear Mechanics 2002; 37(8):1435-1451.

27. Soong TT. Active Structural Control: Theory and Practice. Longman: Essex, U.K., 1990.

28. Chopra AK. Dynamics of Structures. Prentice-Hall:Upper Saddle River, NJ, 2001.

29. Swartz RA, Lynch JP. Partial decentralized wireless control through distributed computing for seismically excited civil structures: theory and validation. American Controls Conference (ACC07), New York, NY, 2007. 